\title{
La Producción Narrativa del MÉdico y EsCritor RAMiro Mendoza Sánchez
}

The Narrative Production of the Physician and Writer Ramiro Mendoza Sanchez

Saniel E. Lozano Alvarado ${ }^{1}$
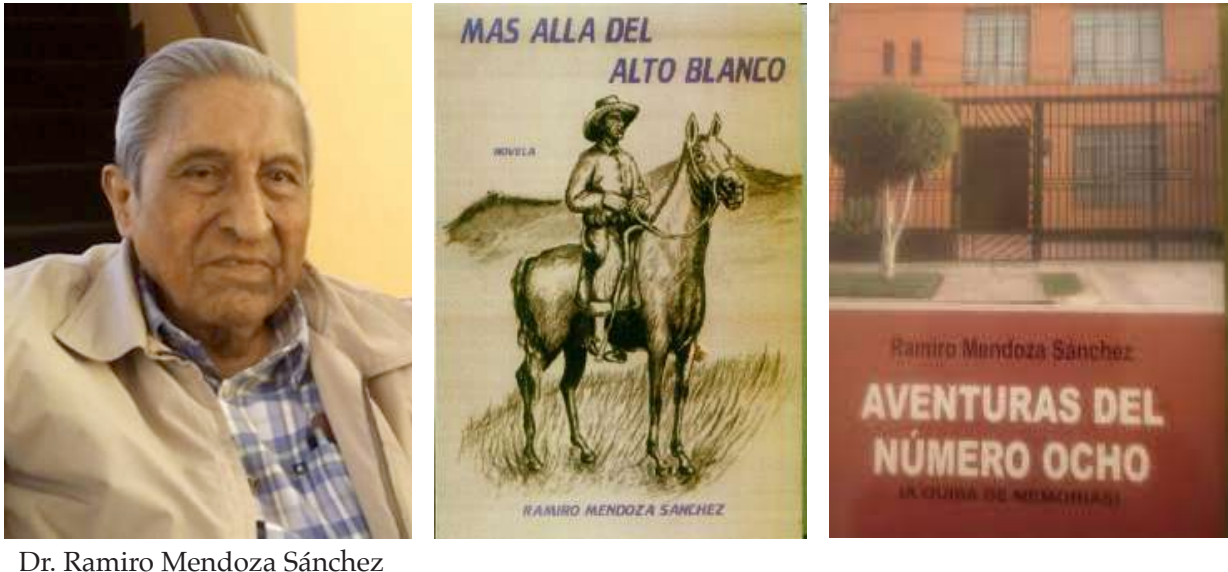

Dr. Ramiro Mendoza Sánchez

\section{INTRODUCCIÓN}

$\mathfrak{E}$

$n$ el presente ensayo ofrecemos un panorama de la producción narrativa del médico y escritor trujillano Ramiro Mendoza Sánchez, nacido el 2 de marzo de 1932 en el distrito de Moche, quien dejó de existir el lunes 30 de octubre de 2017.

El caso no es sorprendente porque, en sentido estricto, no hay oposición entre las profesiones, sean científicas o técnicas y humanísticas, como tampoco puede haber oposición o antagonismo entre las ciencias y las artes. Además, en sentido estricto y limitándonos al ámbito regional, por lo menos en Trujillo tenemos algunos casos ilustrativos de médicos, que al mismo tiempo fueron o son poetas y narradores. Citamos como ejemplo los casos del psiquiatra Erasmo Alayo Paredes y del médico internista contemporáneo Ángel Gavidia Ruiz, ambos de valiosa y reconocida producción.

El trabajo puede ser importante no solo como producto estético sino como tema de reflexión, análisis y valoración en el ambiente artístico, literario, médico y educacional. Por lo tanto, debe constituir especial importancia para los estudiantes y profesionales de educación,

1 Profesor de Apreciación Literaria y Literatura Infantil. Universidad Privada Antenor Orrego (UPAO), Trujillo. Editor científico de la revista de investigación científica "Hampi Runa", UPAO. 
literatura, así como para la comunidad científica y profesional del área médica.

El presente escrito tiene como objetivos:

1. Subrayar la integración entre las ciencias y las artes, especialmente entre la medicina y la literatura; $y$,

2. Analizar la producción narrativa del médico trujillano Ramiro Mendoza Sánchez (1932-2017).

Dada la naturaleza del presente trabajo, la elaboración del presente ensayo se basa y sustenta en el análisis textual, es decir en la lectura exhaustiva de la producción narrativa del autor.

El método que aplicamos, de acuerdo a la naturaleza del trabajo, es fundamentalmente expositivo y argumentativo.

\section{Personalidad Y Trayectoria}

Médico de profesión, dramaturgo, poeta y narrador, hombre de gran prestancia intelectual, de fecunda producción literaria, fue autor de la letra del himno a Trujillo.

Cursó los primeros años de primaria en la Escuela 253, entre el pueblo de Moche, y el verdor de la campiña fresca, eternamente regada por las generosas aguas del río Moche, y el culto encomendado a San Isidro Labrador. Después continuó en el Colegio Nacional de San Juan, donde estudió toda la secundaria. Posteriormente, cursó el ciclo de premédicas en la Universidad Nacional de Trujillo y realizó la carrera profesional en la Escuela de Medicina de San Fernando de la Universidad Nacional Mayor de San Marcos, ambiente recientemente recreado en su última novela, "Las sirenas de Ulises".
Titulado de médico cirujano, se especializó en pediatría, cuyo desarrollo empezó en la Hacienda San Jacinto, provincia del Santa, Ancash; continuó en Chimbote y la culminó en Trujillo, enelHospital Belén. Profesional degran conocimiento y dominio de su especialidad, enriqueció su vida con un fuerte componente sensitivo, social, cultural y artístico. Entonces, no se encasilló ni se aisló en el consultorio sino que se proyectó y desarrolló con otra área también de extraordinaria importancia en su quehacer artístico e intelectual: el ejercicio de la literatura.

Poeta, narrador y dramaturgo de intensa y variada producción, Ramiro Mendoza Sánchez, en el conjunto de su consistente producción, exhibe tres obras narrativas, cuyo contenido nos proponemos presentar.

\section{El Ejercicio Literario}

Pero Ramiro Mendoza no solo ha sido médico sino que, con igual pasión, se entregó a las letras, que las cultivó simultáneamente con el desempeño de la función médica. En efecto, su compromiso con la creación literaria ha brotado en varios títulos, en cuyo conjunto ha publicado, en poesía: "Antología poética" y "110 poemas de amor"; en novela: "Más allá del Alto Blanco", "Los muchik de mi valle" y "Las sirenas de Ulises". Igualmente, en teatro es autor de dos volúmenes de "Gentes de mi tiempo", que comprende comedias ("Se necesita muchacha", "El estuche de Pandora", "iYo me quemo las pestañas!", "Un panal de rica miel"), drama ("Lesbia"), tragedia ("Después del banquete") e himnos de varias ciudades e instituciones.

\section{La Producción Narrativa}

En esta parte desarrollamos un panorama de su producción literaria manifestada específicamente en la novela, que comprende 
los volúmenes "Más allá del Alto blanco" y "Las sirenas de Ulises", así como el relato testimonial, a modo de memorias: "Aventuras del número ocho".

\section{a. "Más allá del Alto blanco"}

Es su primera novela, aparecida en 1999. Constituye una hermosa y tierna historia de amor cuyos protagonistas -Andrés y Elena- se conocen desde niños en las fértiles tierras de la campiña de Moche, principal escenario de los acontecimientos.

Junto a la historia principal, o junto a ella, se desarrolla otra relación amorosa, la de Hildaura y Celedonio, inmigrantes andinos procedentes del sur del país, quienes se incorporan a la campiña de Moche, sin perder su identidad nativa, con lo cual el narrador proyecta una cobertura de implicancias nacionales.

Asimismo, el amor entre los protagonistas no se desarrolla con plena armonía sino que se ensombrece por una serie de vicisitudes, principalmente por el sentimiento enfermizo que experimenta una muchacha lesbiana por la heroína de la novela.

Este eje narrativo, que da unidad a la novela, permite al narrador desarrollar su pensamiento en torno a la oposición entre el pasado y el presente, entre la tradición y la modernidad. En esa oposición, el autor no puede evitar sus sentimientos y su adhesión por los ancestros nativos, por la vida del pueblo, por la vida sencilla del campo, al mismo tiempo que una fuerte crítica la dirige al ambiente de la ciudad, a los signos de la modernidad y a los riesgos que permanentemente tienen que enfrentar los jóvenes. Entonces, todo el conjunto de la obra se revela como un animado y vivo cuadro de tradiciones, costumbres, creencias, supersticiones, folclore, religiosidad, plenitud del amor, conflicto de pasiones, exaltación de la vida agraria y aldeana o pueblerina, lucha contra el abuso de las autoridades y de los acaparadores de las tierras, en contra del campesinado.

Según el tema expuesto, los protagonistas pertenecen al estrato popular y campesino; por tanto, el lenguaje es sencillo, coloquial, sin galanuras pero con una técnica de construcción lineal, atractiva y de creciente y permanente atracción.

\section{b. "Aventuras del número ocho"}

Constituye su segunda obra, en la que consciente y deliberadamente se aparta del mundo de la ficción para ofrecernos un relato testimonial y vivencial, a modo de memorias y confesiones testimoniales. Desde luego, el título es novedoso y sorprendente; pero, para que no quepan mayores dudas o especulaciones, mágicas o esotéricas, el mismo autor aclara:

"Sin meterme en esoterismos, en los cuales soy un perfecto ignorante, y sin dejarme arrastrar por supersticiones capaces de desviar mi camino; observando simplemente los variados aspectos de mi anodina trayectoria por el mundo y los de mis características personales y familiares, he llegado a comprobar que mi factor es el número ocho. Si esto es bueno o malo para mí, no lo sé. Si influye en la misión que, como le ocurre a todo el mundo, he traído para cumplir de buen o mal grado, no tengo idea" (p. 7).

A continuación, a manera de ejemplo y comprobación de su declaración, cita estos párrafos:

Tengo 8 hijos, con la certeza de que no podré tener más, entre otras razones, por mi avanzada edad y por la fidelidad que ahora guardo para con 
mi esposa. Mi nombre completo consta de 8 silabas.

Cuando llegué al mundo, mi Villa natal contaba con 8 calles de este a oeste y 8 de norte a sur. Las primeras eran More, Inclán, Carlos de los Heros, Bolognesi, Grau, O'Donovan, Salaverry y Elias Aguirre (donde nací). Las otras, Sepúlveda, Diego Ferré, Leoncio Prado, Ladislao Espinar, Alfonso Ugarte, Jorge Chávez, San Martín y José Gálvez.

En las páginas que siguen, el amable lector podrá darse cuenta fácilmente de cómo mi existencia se ha desarrollado en periodos bien definidos, de 8 años cada uno, sin que yo los hubiera calculado, previsto o deseado. ( $p .7)$

La estructura y organización del texto, efectivamente, se distribuye a lo largo de ocho capítulos, cada uno de los cuales, con excepción del último, que es el más extenso, comprende, a su vez, ocho temas específicos. La mayor parte de los títulos no son directos, declarativos o transparentes, sino más bien sugerentes, emotivos, plásticos y metafóricos: La semilla en el surco, Riegos y cultivos del vástago, El fruto en sazón, El polen a merced del viento, Los renuevos del arbusto, Los afanes del horticultor, Realización vocacional y La cesantía.

Cada capítulo, a su vez, con excepción del último, que es el más extenso, está compuesto de ocho temas específicos. Citamos como ejemplo el capítulo IV. El polen a merced del viento, cuyo contenido se desarrolla a través de los siguientes puntos: El internado; En busca de mis destinos; Un año crucial y maravilloso: 1959; Mi primer empleo médico; Mi ingreso al Ministerio de Salud; Mis pininos teatrales; Mis tres hijos chimbotanos; Mi labor médica en Chimbote; $y$, Dramaturgo y Director de Club teatral.

Con gran capacidad de evocación, nostalgia y también recreación y recuperación del tiempo y la memoria; con una prosa límpida y tersa; con un lenguaje declarativo y sencillo; con un estilo coloquial y con un sentido afirmativo de la vida, el escritor nos ofrece un vivo recuento y recorrido de su vida en el pintoresco pueblo de Moche, su tierra natal y también tierra de sus ancestros; sus correrías por el pueblo y la campiña; sus estudios primarios allí mismo, en su pueblo natal; los secundarios, en el Colegio Nacional San Juan y los universitarios en la Facultad de Medicina de San Fernando.

En lo que se refiere al contenido, una frondosa galería de sus amigos, compañeros de barrio, de las aulas, de sus maestros; sus inquietudes culturales, artísticas, sociales y deportivas; sus escarceos enamoradizos; su plenitud familiar y conyugal; la vida pletórica en su hogar y el recuento cariñoso de su descendencia que menciona con orgullo y amor.

También el importante libro nos muestra un recorrido intenso del autor en su desempeño profesional desde los comienzos tentativos y difíciles hasta su afirmación por la antigua Hacienda San Jacinto, Chimbote y Trujillo. Una vida matizada, combinada y enriquecida con los continuos hechos sociales y culturales, las aficiones por el baile y la música; las anécdotas y diversiones; los viajes turísticos por diversos lugares de nuestra patria, como el Callejón de Huaylas y Machu Picchu; pero, también el dolor y la tragedia, la proyección social y la solidaridad brotadas, de manera especial, a raíz del terrible terremoto de 1970, que lo vivió precisamente en el propio puerto chimbotano. Habiendo vivido la hecatombe del terrible movimiento sísmico en el propio núcleo de la zona devastada, ofrece su propio testimonio:

"Aquel día había salido yo de vacaciones en el Centro de Salud. El domingo 31 de mayo amaneció con cielo claro y despejado. En México iba a iniciarse el Campeonato Mundial de Fútbol y Perú participaba en el primer partido, el inaugural. En la urbanización Buenos Aires 
nos reunimos varias parejas de amigos vecinos, para almorzar y ver por televisión el partido en casa de Cono Nicosia. A eso de las once el cielo se oscureció de tal modo que nos llamó la atención. Nuestros niños jugaban en las calles circundantes.

Nos habiamos sentado a la mesa, a degustar un sabroso cebiche, como inicio del almuerzo. De pronto, se produjo un ruido espantoso. Parecía que golpeaban las paredes con gigantescos mazos. El piso tembló violentamente. Salimos todos a la calle, corriendo. Buscábamos a nuestros hijos. Eran las 3 y 23 minutos de la tarde. El piso asfaltado de las calles se abría en zanjas que parecian culebras deslizándose rápidamente. Fueron treinta segundos que parecieron interminables. La confusión era grande. Las paredes de las casas se rajaban. Algunas se cayeron. Pasado el primer remezón, logramos reunir a los niños, que estaban aterrorizados. Pedrito, mi hijo de cinco años, se arrodilló y mirando al cielo pidió piedad a Dios. Los movimientos denominados réplicas se sucedian cada cinco, diez o quince minutos. Habiamos perdido totalmente el apetito; ni siquiera pude tragar yo un sorbo de agua que me ofreció mi esposa. Alguien me avisó que al frente, en el pueblo joven Villa María, de casas de adobe y terreno pantanoso, había muertos y heridos. Mi condición de médico me exigía ir de inmediato y fui al puesto de salud de esa barriada. Los cadáveres empezaban a ser recogidos en la plataforma de un camión. Los heridos necesitaban atención. Los botiquines estaban cubiertos de polvo y escombros. Todos ayudaban. La carretera panamericana se habia abierto en una gran zanja que interrumpió el tráfico. Supimos que Chimbote, ciudad antigua, de construcciones de adobe y quincha, se habia destruido casi totalmente. Alguien nos dijo que el terremoto había abarcado el Callejón de Huaylas y, en la costa, hasta cerca de Lima por el sur, y más allá de Trujillo por el norte. Que
Moche había quedado en ruinas y con muchos muertos.

Por la noche, a merced de tablones colocados a modo de puentes sobre la zanja, pudimos pasar a Chimbote. No habia luz. La destrucción era increible. Pronto se supo que Yungay había desaparecido totalmente, sepultada por un aluvión proveniente del Huascarán y que habia miles de muertos. Trujillo, Huaraz, Nepeña, Casma, estaban sumidos en escombros. Se llegó a calcular en 70,000 los muertos en toda la zona afectada. Los días que siguieron fueron de titánicos esfuerzos para la reconstrucción en lo que permitía la emergencia. Logré viajar con mi pequeña familia por un día a Moche, donde supe que felizmente no se había registrado desgracia en mi parentela.

De inmediato comenzó la tarea de reconstrucción en todos los sectores: salud, educación, servicios públicos. El norte del país estaba de luto. No habia trabajo. Los temblores siguieron durante muchos días y se temía la inundación desde el océano. El Centro de Salud donde trabajaba, desapareció. Todos fuimos trasladados al Hospital de La Caleta, donde asumíla jefatura de Pediatría. Estuvimos atendiendo en carpas, en previsión de epidemias. De todos modos se presentaron las acarosis, las enterocolitis, las bronconeumonías y la desnutrición consecuente a la falta de la población. La ayuda extranjera se hizo presente desde el primer momento: Chile y Cuba fueron los primeros. (pp. 95 y 96).

Aparte de este testimonio personal, el autor desarrolla con gran despliegue de vivencias, testimonios y experiencias otros hechos igualmente importantes y valiosos, especialmente los que se relacionan con el ejercicio médico, en cuyo conjunto destacamos estos hechos: el internado, mi primer empleo médico, mi ingreso al Ministerio de Salud, mi labor médica en Chimbote. 
Posteriormente, con ocasión de su traslado a Trujillo, el autor explica los cargos, funciones, tareas y actividades propias de la función médica. Así lo indican, específicamente los temas El Consejo Regional I del Colegio Médico del Perú, Mi regreso al Hospital de Belén, Mi reinserción hospitalaria, La Medicina Integral.

Asimismo, el autor otorga especial relieve a determinados hechos médicos, como se puede apreciar a través del desarrollo de los siguientes temas: Mis colegas del Departamento de Pediatría, La pasantía de los internos, El Hospital de Belén a su comunidad. En esta misma destaca la personalidad y trascendencia de la función profesional desempeñada por importantes médicos liberteños, como los doctores Alejandro Falcón, Gilberto Alvarado, Julio Mendiburu Velit, Francisco San Martín. De manera muy especial destaca el autor el Discurso de orden que pronunció el 5 de octubre de 1983 en la ceremonia organizada por el Consejo Regional I del Colegio Médico del Perú, presidido por el doctor Alamiro Vallejos en homenaje a Daniel Alcides Carrión y a la Medicina Peruana en su día. La exposición fue muy elogiada y comentada por los participantes a la respectiva ceremonia. El orador concluyó con las siguientes palabras:

"La Promoción fernandina de 1957, a la que pertenezco y lleva el nombre del Centenario Carrión, porque el 13 de agosto de ese año se conmemoraba el primer centenario del nacimiento de héroe, me permite expresar con orgullo las palabras finales: Honor y gloria a la Medicina Peruana en la egregia figura de Carrión; honor a la Medicina Peruana en la persona de los grandes maestros que han producido nuestras universidades; honor a la Medicina Peruana en el recuerdo de tantos héroes anónimos que consciente o inconscientemente siguieron los pasos de ese hombre gigantesco que nació humildemente en una aldea de Cerro de Pasco, creció en el ámbito nacional y se universalizó hasta trasponer los linderos donde comienza la inmortalidad. He dicho." (pp. 123 y 124).

Como intelectual y médico, Ramiro Mendoza Sánchez es también pródigo en el recuerdo de los amigos, compañeros, jefes y autoridades culturales, sociales y profesionales. Un espacio importante dedica a la consolidación de la familia, el amor y los hijos, a lo cual agrega y profundiza la fe y conciencia en el curso de la vida; sus convicciones en la grandeza y valor del arte;

De esta manera, el distinguido autor no solo enriquece su producción bibliográfica, sino que escribe desde la cima del espíritu y de la vida, sin amarguras ni resentimientos, sin lamentaciones ni frustraciones, sino con la confianza y gratitud a la existencia. Por eso nos adherimos fraternalmente al brote de este nuevo fruto intelectual que consolida una meritoria trayectoria creadora.

\section{c. "Las sirenas de Ulises"}

El tema central refiere cómo un estudiante de medicina, quien había estudiado la secundaria en el Colegio Nacional de San Juan, de Trujillo, pero que se forma profesionalmente en la Facultad de Medicina de San Fernando, vive una intensa experiencia amorosa plasmada en relaciones íntimas principalmente con la coprotagonista, y también con otras parejas menos comprometidas pero igualmente enamoradas e ilusionadas con el entonces estudiante y futuro médico.

Este hecho, sin embargo, no llega a poner en riesgo el objetivo principal que motivó al protagonista ir a estudiar en Lima con el apoyo de sus padres, radicados en Moche, aunque, por supuesto, hay escenas en los que parecieran entrar en conflicto los estudios o el amor, no obstante que Ulises es consciente 
de su objetivo primordial de titularse como médico, tras cuya búsqueda refiere también los trajines, estudios, prácticas y exámenes que debe cumplir como estudiante, así como las angustias, preocupaciones y peripecias de estudiante provinciano.

La trama de la novela se desarrolla a través de dos ejes argumentales: el principal y nuclear es el amor, con el cual alternan las inquietudes, vivencias y experiencias de los estudios profesionales, hasta que, una vez titulado, el protagonista retorna a su pueblo natal de Moche para iniciar el ejercicio de su carrera profesional en la especialidad de pediatría. En Lima quedan su amada y otros entretenimientos.

En cuanto al ambiente, sobre el trasfondo de una rica y consistente cultura general, en cuyo conjunto la literatura es un componente primordial, el título remite a reminiscencias antiguas y clásicas; sin embargo, tal título no deja de ser un pretexto, porque el ambiente que se vive es de clara y explícita recreación de elementos, factores, signos y expresiones de la cultura peruana: urbana, criolla, tradicional y popular. De manera específica se destacan dos escenarios: Lima y el pueblo de Moche.

En lo que se refiere al manejo de recursos artísticos y expresivos, admira el empleo de una arquitectura verbal de esmerado nivel, lo cual revela, a su vez, solvencia y oficio literario y narrativo. De esta manera, resulta evidente el manejo de una técnica narrativa sostenida en un eje de continuo y permanente interés, que aumenta la expectativa por la sucesión de los hechos futuros. El dominio de estos aspectos revela claramente que el escritor trujillano ha llegado a convertirse en un escritor de consistente, continua y fecunda producción.

Sobre las motivaciones y fuentes de producción de la novela, el autor no lo declara pero es fácil deducir la presencia del componente autobiográfico, por lo que el protagonista Ulises es, indiscutiblemente, el alter ego del autor, es decir, su otro yo. Por eso la obra, por más que sus personajes se escondan bajo otros nombres, y al margen de que la obra tenga elementos de ficción, lo que le otorga carácter y consistencia es su naturaleza y carácter realistas. Al fin de cuentas, aparte de las motivaciones o caracteres específicos, de lo que se trata es de reconocer el valor de esta nueva novela, que enriquece sustancialmente el proceso de la literatura regional de modo preponderante. Por tanto, hay que estimular y promover el acceso a ella.

\section{Conclusiones}

Primera. El médico Ramiro Mendoza Sánchez alternó su profesión con el ejercicio de la literatura.

Segunda. En literatura, el autor cultivó varios géneros, especialmente narración, teatro $\mathrm{y}$ poesía.

Tercera. En narración, la producción se ha volcado en dos novelas y un libro de memorias. Cuarta. La obra narrativa del autor es fuertemente testimonial, con frecuencia se sustenta en el desarrollo de aspectos autobiográficos; es decir, la literatura que cultiva, con frecuencia no es de ficción sino realista.

Quinta. En el cultivo de la literatura, el autor revela un respetable nivel del lenguaje artístico

\section{REFERENCIAs BibliográficAs}

1. Mendoza Sánchez, Ramiro

1999. Más allá del Alto blanco. Chiclayo: edición del autor. 2008. Gentes de mi tiempo (teatro). Trujillo: Industria Gráfica Libertad S.A.C.

2016. Aventuras del número ocho (A guisa de memorias). Trujillo: Industria Gráfica Libertad S.A.C.

2017. Las sirenas de Ulises. Trujillo: Industria Gráfica Libertad S.A.C. 